\title{
RELATIONS ENTRE LES CARACTÉRISTIQUES DE L'HABITAT ET LES POPULATIONS DE TRUITES COMMUNES (SALMO TRUTTA L.) DE LA VALLÉE DE LA NESTE D'AURE.
}

\author{
P. BARAN, M. DELACOSTE, J.M. LASCAUX, A. BELAUD.
}

Laboratoire d'Ingénierie Agronomique, Equipe Environnement Aquatique et Aquaculture, ENSAT, 145 Av. de Muret, 31076 TOULOUSE Cedex, France.

\section{RÉSUMÉ}

Les relations entre les caractéristiques de l'habitat et les biomasses et densités de truites communes (Salmo trutta L.) ont été recherchées dans 33 stations de la rivière Neste d'Aure et trois de ses affluents : la Neste du Louron, la Neste du Rioumajou et le ruisseau d'Espiaube dans le département des Hautes-Pyrénées. L'étude a été conduite sur un cycle annuel. Dans un premier temps, la validité du modèle d'Indice de Qualité d'Habitat (HQI) (BINNS et EISERMAN, 1979), basé sur 10 variables de l'habitat, a été testée. Les biomasses théoriques prévues par le modèle ne sont pas linéairement corrélées aux biomasses observées par pêche électrique. Le meilleur ajustement linéaire est obtenu grâce à des transformations par les logarithmes. Toutefois, la pente de la droite de régression est significativement différente de $1(t=2.53(p<0.01))$. Le modèle de l'Indice de Qualité d'Habitat ne constitue pas, dans le cas de la vallée d'Aure, un outil satisfaisant de prévision des biomasses de truites. Dans un deuxième temps, l'influence de chaque variable de l'habitat a été testée individuellement. Les biomasses observées sont significativement corrélées à l'altitude (entre 1350 et $600 \mathrm{~m}$ ), aux surfaces d'abris, à la température mensuelle maximale (pour une gamme allant de 10 à $16^{\circ} \mathrm{C}$ ), à la conductivité électrique, à la vitesse moyenne au fond, à la profondeur moyenne et au rapport largeur/profondeur. Les densités sont significativement corrélées aux mêmes variables, à l'exception de là profondeur moyenne; il faut également ajouter des corrélations significatives avec la pente de la ligne d'eau et la largeur de la rivière. L'étude par classe d'âge montre que l'abondance de la cohorte $0+$ est liée à l'altitude, la température et la conductivité. La largeur moyenne constitue la seule caractéristique de l'habitat physique corrélée avec les biomasses et densités de $0+$. L'étude par saison indique seulement une corrélation négative entre les densités et biomasses échantillonnées en hiver et la profondeur moyenne. En ce qui concerne la cohorte $1+$, on observe des corrélations avec les mêmes variables altitude, température et conductivité auxquelles ii faut ajouter la variable abris. Les densités de truites de taille supérieure à la taille légale de capture $(180 \mathrm{~mm})$ sont positivement corrélées à la surface d'abris, la profondeur moyenne, la température et la conductivité, et négativement avec l'altitude. Dans une troisième étape, à partir de régressions multiples progressives, il a été possible d'établir un modèle statistique à 5 variables qui explique $86 \%$ de la variation de biomasse totale de truites.Ce type d'outil peut constituer un élément de gestion pour les populations de truites de la Vallée de la Neste d'Aure.

Mots-clés : Truite commune, Salmo trutta, habitat, corrélation, biomasse, densité, classe d'âge. 


\title{
RELATIONSHIPS BETWEEN HABITAT FEATURES AND BROWN TROUTS POPULATIONS (SALMO TRUTTA L.) IN NESTE D'AURE VALLEY.
}

\begin{abstract}
The relationships between habitat features and biomass and densitiy of brown trout (Salmo trutta L.) were studied in 33 sections of the Neste d'Aure stream and three of its tributaries: the Neste du Louron, the Neste du Rioumajou and the Espiaube stream in the Hautes-Pyrénées region. The Habitat Quality Index (HQI) model I (BINNS and EISERMAN, 1979 ), based on 10 environmental variables, was tested. The biomass predicted by the model were not linearly related with biomass of trout observed by electrofishing on the 33 sections. The best linear model was obtained after logarithmic transformations of the two variables. However, the slope of the regression line was significantly different from $1(t=2.53$ $(p<0.01))$. The HQl model did not appear to be a good method of assessing the biomass of brown trout in the Neste d'Aure valley. Correlations between habitat variables and brown trout biomass and density were investigated. Total biomass was significantly related to elevation (between 1350 and $600 \mathrm{~m}$ ), cover, maximum summer temperature (between 10 and $16{ }^{\circ} \mathrm{C}$ ), conductivity, mean bottom velocity, mean depth and width/depth ratio. Total density was significantly related to the same variables, with the exception of mean depth, and in addition to water gradient and stream width. The study by age-class showed that the abundance of young-of-the-year trout is related to elevation, temperature and conductivity. Only width was the habitat feature related to abundance of young-of-the-year. No correlations were found with other physical habitat features. Abundance of one-year-old trout $(1+)$ was related with the same variables of elevation, temperature and conductivity in addition ; their density and biomass were also related to cover. Density of catchable trouts (length greater than $180 \mathrm{~mm}$ ) was correlated with cover, depth, temperature, elevation and conductivity. The stepwise regression analyses produced combinations of variables that explained $86 \%$ of the variations in biomass (with 5 variables). This type of work can be very useful in the management of fishing and of trout populations.
\end{abstract}

Key-words : Brown Trout, Salmo trutta, habitat, correlation, biomass, density, age-class.

\section{INTRODUCTION}

La truite commune (Salmo trutta L.) possède des exigences strictes vis-à-vis de son environnement (LEWIS, 1969). La nature et l'influence relative de ces facteurs de l'habitat évoluent selon le stade de développement (HAURY et al., 1991). Les variables les plus couramment citées se rapportent à l'habitat physique, la physico-chimie de l'eau et à la faune benthique. L'échelle d'analyse des relations entre les populations de truites et leur environnement constitue également un élément déterminant (HAURY et al., 1991). Certaines variables auront une influence à l'échelle du microhabitat, d'autres à l'échelle du faciès d'écoulement (macrohabitat) ou de la séquence de faciès d'écoulement.

De nombreuses investigations ont cherché à corréler la densité ou la biomasse de truites avec l'habitat physique ou les caractéristiques de débit. FAUSH et al. (1988) recensent 70 modèles permettant d'estimer l'abondance des Salmonidés à partir des variables de l'habitat. On peut citer, parmi eux, les modèles proposés par CUINAT (1971), PHILIPPART (1978) in WELCOMME (1985), BINNS et EISERMAN (1979), RALEIGH et al. (1986), LANKA et al. (1987). La méthode de l'Indice de Qualité d'Habitat (HQI) (BINNS et EISERMAN, 1979) retient 10 variables de l'habitat. Ses applications dans la littérature sont nombreuses (EIFERT et WESCHE, 1982 ; CONDER et ANNEAR, 1987 ; BOWLBY et ROFF, 1986 ; SCARNECCHIA et BERGERSEN, 1987 ; HARRIS et al., 1991). La diffusion d'un manuel d'utilisation (BINNS, 1982) permet l'application standardisée de cette méthode.

La présente étude, conduite de septembre 1990 à janvier 1992, s'est fixée pour premier objectif de tester la validité du modèle HQl sur une population de truites communes des Pyrénées. Le test de validité de ce type de modèle ne peut être envisagé que sur un milieu répondant à deux conditions : ne pas avoir subi d'événements de pollution 
accidentelle récents et ne pas se situer en limite aval de la zone à truites. La Neste d'Aure répondait à ces critères et offrait une variabilité et une représentativité des données d'habitat conformes avec les objectifs de l'étude. Les principaux facteurs influençant la biomasse et la densité de poissons ont également été recherchés, tant du point de vue qualitatif que quantitatif. La mise au point d'un outil de calcul des biomasses et densités de truites pour la rivière considérée a constitué la dernière étape de ce travail.

\section{PRÉSENTATION DU SITE (figure 1)}

La Neste d'Aure, affluent le plus occidental de la Garonne pyrénéenne, est formée de la confluence de deux ruisseaux à 1100 m d'altitude : la Neste de Couplan et la Neste d'Aragnouet. Les stations d'étude se situent sur le secteur amont de la rivière, ainsi que sur les trois principaux affluents dans ce secteur : la Neste du Louron, la Neste du Rioumajou et le ruisseau d'Espiaube. Le régime hydrologique de ces rivières est de type nival (étiage hivernal). Chacune peut être séparée en secteurs écologiques, dont les caractéristiques sont présentées dans le tableau l.

Tableau I : Principales caractéristiques des rivières étudiées.

Table I : Main characteristics of streams studied.

\begin{tabular}{|c|c|c|c|c|c|c|}
\hline Rivières & $\begin{array}{c}\text { Secteurs } \\
\text { écologiques }\end{array}$ & $\begin{array}{l}\text { Altitude } \\
\text { (m) }\end{array}$ & $\begin{array}{c}\text { Largeur } \\
\text { moyenne } \\
(m)\end{array}$ & $\begin{array}{c}\text { Pente } \\
\text { moyenne } \\
(\%)\end{array}$ & $\begin{array}{c}\text { Module } \\
\mathrm{m}^{3} / \mathrm{s}\end{array}$ & $\begin{array}{c}\text { Longueur court-circuitée par } \\
\text { des installations hydró́lectriques } \\
\text { (kms) }\end{array}$ \\
\hline \multirow{3}{*}{ NESTE D'AURE } & 1 & 1017 à 810 & 8 & 3.6 & 9.2 & \multirow{3}{*}{14.4} \\
\hline & ॥ & 810 à 670 & 17 & 1.1 & 20.3 & \\
\hline & III & 670 à 630 & 15 & 2 & 22.5 & \\
\hline \multirow[b]{2}{*}{ NESTE DU RIOUMAJOU } & I & 1560 à 1350 & 8 & 3 & 2.0 & \multirow[t]{2}{*}{5.5} \\
\hline & II & 1350 a 1005 & 6.5 & 8.1 & 2.5 & \\
\hline \multirow{5}{*}{ NESTE DU LOURON } & 1 & 1200 à 1100 & 6 & 2 & 0.3 & \multirow{5}{*}{12.0} \\
\hline & II & 1100 a 1005 & 8 & 0.8 & 0.4 & \\
\hline & III & 1005 a 980 & 12 & 2.6 & 3.6 & \\
\hline & IV & 980 à 890 & 12 & 0.7 & 4.3 & \\
\hline & v & 890 a 700 & 7 & 1.8 & 1.7 & \\
\hline \multirow[b]{2}{*}{ RUISSEAU D'ESPIAUBE } & 1 & 1100 \& 850 & 3 & 5 & 0.4 & \multirow[b]{2}{*}{3.0} \\
\hline & $\|$ & 850 a 800 & 2.5 & 2 & 0.1 & \\
\hline
\end{tabular}

\section{MÉTHODOLOGIE}

\subsection{Techniques d'échantillonnage}

\subsubsection{Données relatives aux populations de truites}

La pêche électrique a été pratiquée à l'aide d'un groupe électrogène fixe, de type Electro-Pullman (EPMC), délivrant un courant électrique sous une tension de 480 à 600 Volts pour une intensité de 1 à 2 Ampères. Sur chacune des 33 stations; plusieurs passages successifs ont été effectués à effort de pêche constant conformément à la méthode de De Lury (DE LURY, 1951). Les poissons ont été pesés à $1 \mathrm{~g}$ près et mesurés à $1 \mathrm{~mm}$ près (longueur totale).

Dans 27 des 33 stations, les pêches électriques ont été réalisées à quatre reprises afin de calculer la variation intra-stationnelle d'abondance des truites. Six stations n'ont pu être échantillonnées qu'une ou deux fois en période de débit d'étiage en raison de problèmes d'accès.

\subsubsection{Données relatives aux caractéristiques du milieu}

Les caractéristiques de l'habitat physique, la physico-chimie de l'eau ainsi que la faune d'invertébrés benthiques ont été mesurées sur les 33 stations. Les 10 variables utilisées dans le modèle de calcul de la méthode HQI ont été échantillonnées selon le protocole indiqué par BINNS (1982) en période de débit d'étiage. 
Bull. Fr. Pêche Piscic. (1993) 331 : 321-340 - 324 -

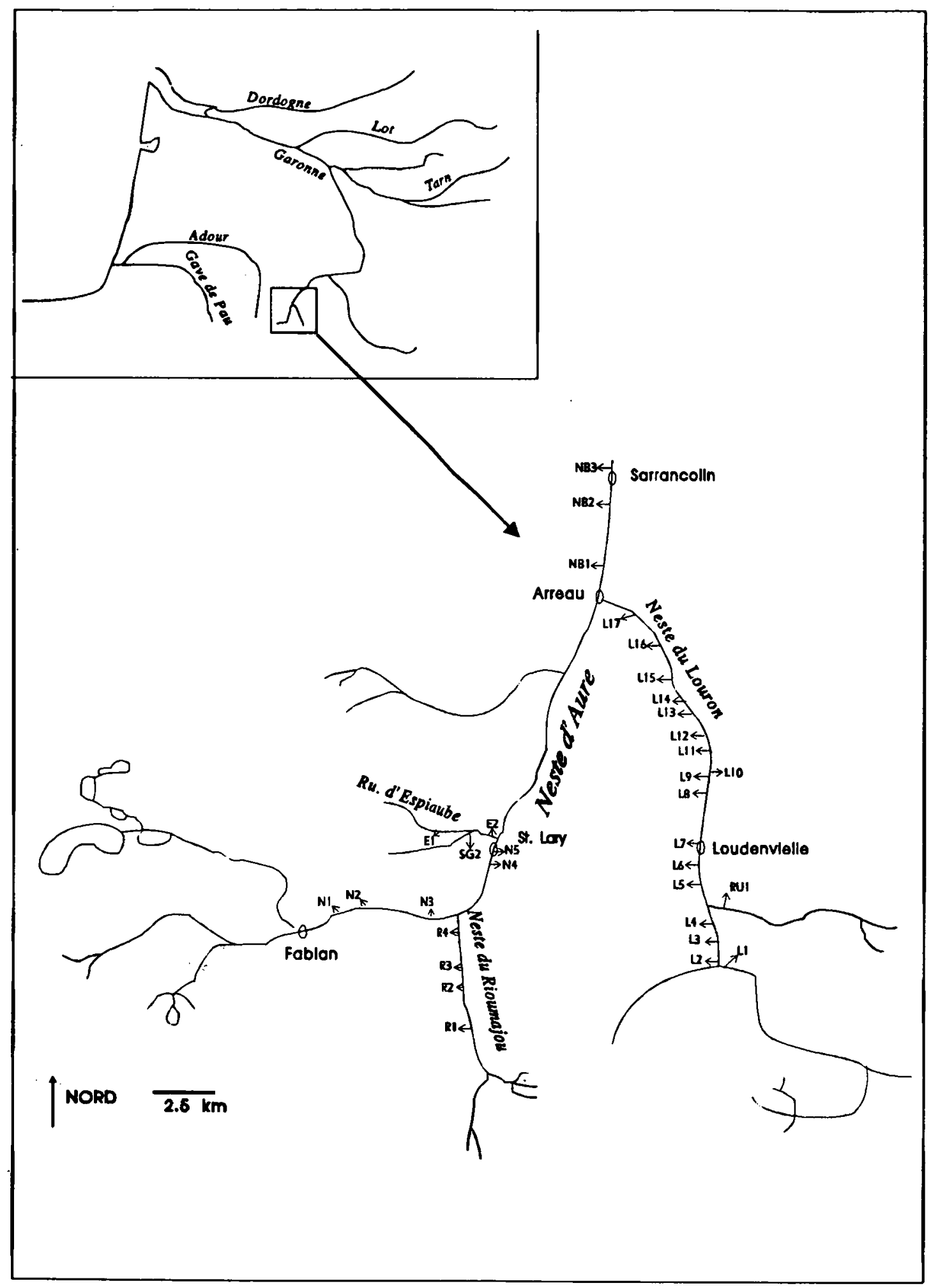

Figure 1 : Situation géographique.

Figure 1: Geographical situation. 


\section{Variables de la méthode HQI}

- La température mensuelle maximale ou température moyenne du mois le plus chaud correspond à la moyenne des températures relevées en chaque station grâce à des thermomètres mini-maxi durant les mois de juillet, août et septembre.

- Les débits ont été obtenus à partir des enregistrements de 5 limnigraphes (annuaire des débits en Midi-Pyrénées, SRAE), et complétés par des données EDF-DTG Ressources en Eau et des mesures ponctuelles à la perche pyrée. Le rapport du module annuel sur le débit moyen d'étiage ainsi que le rapport du débit maximal journalier sur le débit minimal journalier ont été calculés. Comme indiqué par BINNS (1982), la moyenne des rapports calculés sur les cinq dernières années d'enregistrement a été utilisée dans le modèle.

- La concentration en nitrate a été dosée (à $0,1 \mathrm{mg} / \mathrm{l}$ près) en période estivale par colorimétrie.

- La vitesse de surface a été calculée en chronométrant le temps d'écoulement d'un colorant .

- L'abondance et la diversité du peuplement d'invertébrés ont été calculées à partir des tris et des déterminations de prélèvements effectués en période estivale à l'aide d'un filet surber sur des faciès lotiques et lentiques de chaque station.

- Les abris : ARNETTE (1976) in BINNS et EISERMAN (1979) définit les abris comme toute zone dans le chenal permettant à la truite de se réfugier et de se dissimuler des prédateurs. Cette définition permet de considérer comme abris à la fois des zones ayant une hauteur d'eau suffisante, des zones turbulentes, des gros blocs, tout élément submergé, les affouillements en sous-berges ou encore la végétation aquatique. Leur utilisation par le poisson varie en fonction de la saison et surtout en fonction de la taille des individus (BUTLER et HAWTHORNE, 1968). En suivant le protocole de mesure des abris établi par BINNS (1982), la longueur et la profondeur des sous-berges, des sous-blocs, des enrochements et des branchages ainsi que la surface des zones profondes et des zones turbulentes ont été mesurées. Le pourcentage de surface d'abris a été ensuite calculé en effectuant le rapport de la surface occupée par les abris sur la surface totale de la station.

- La longueur des rives subissant l'érosion par le courant a été mesurée.

- La largeur moyenne a été calculée à partir de transects transversaux (en moyenne, 1 tránsect tous les $6 \mathrm{~m}$ ).

\section{Variables complémentaires}

En complément de ces variables, 4 autres paramètres ont été déterminés sur chaque station. II s'agit :

- de la vitesse au fond à l'aide d'un courantomètre March Mac Birney modèle 201 $D$ (précis à $1 \mathrm{~cm} / \mathrm{s}$ ). Les mesures ont été effectuées le long de transects, avec un pas variant de 0,1 à $1 \mathrm{~m}$ selon la largeur de la station. Chaque transect a été choisi comme représentatif d'une zone où les conditions morphodynamiques sont homogènes. En moyenne, un transect tous les $6 \mathrm{~m}$ de rivière a été effectué.

- de la profondeur sur les mêmes transects que la vitesse au fond,

- de la pente de la ligne d'eau au goniomètre,

- de la conductivité électrique (moyenne des mesures sur quatre campagnes).

\subsection{Traitement des résultats}

Les biomasses et densités de truites ont été estimées, pour chaque station, à partir de la méthode de De Lury (LAURENT et LAMARQUE, 1975). Les fréquences de classes de tailles ainsi qu'une étude scalimétrique ont permis de calculer les abondances respectives des classes d'âges $0+$ et $1+$. Pour la classe d'âge $0+$, seuls les résultats des campagnes d'été, d'hiver et d'automne ont été pris en compte. En effet, la faible taille des individus au printemps limitent leur capturabilité par la pêche électrique et entraîne ainsi un biais important dans l'échantillonnage. 
Les relations entre les caractéristiques physiques du milieu et l'abondance numérique et pondérale des truites ont été examinées à partir de plusieurs techniques de corrélation et de régression. La normalité de la distribution conjointe des couples de variables aléatoires utilisées dans l'analyse a été testée à partir du test de Kolmogorov-Smirnov. Lorsque celle-ci a été validée, des régressions simples ont été réalisées. La validité des modèles linéaires obtenus a été testée à partir de l'examen des résidus réduits (TOMASSONE et al., 1983). Lorsque les hypothèses d'indépendance et de normalité de la distribution des fréquences relatives cumulées des résidus réduits ont été rejetées, des transformations de variables ont été effectuées afin d'obtenir le meilleur ajustement linéaire. Lorsque l'hypothèse de normalité de la distribution conjointe des couples de variables aléatoires a été rejetée, la méthode non-paramétrique du coefficient de corrélation de rang de Spearman (rs) a été utilisée (SPRENT, 1992). Les techniques de régressions multiples progressives (DAGNELIE, 1975 ; TOMASSONE et al., 1993) ont été appliquées. L'examen du coefficient de corrélation multiple et des résidus réduits a permis de valider cette approche.

Disposant de données sur le peuplement d'invertébrés, le modèle $n^{\circ} 1$ de la méthode de l'Indice de Qualité d'Habitat a été appliqué selon le protocole indiqué par BINNS (1982).

Modèle $\mathrm{n}^{\circ} 1$ :

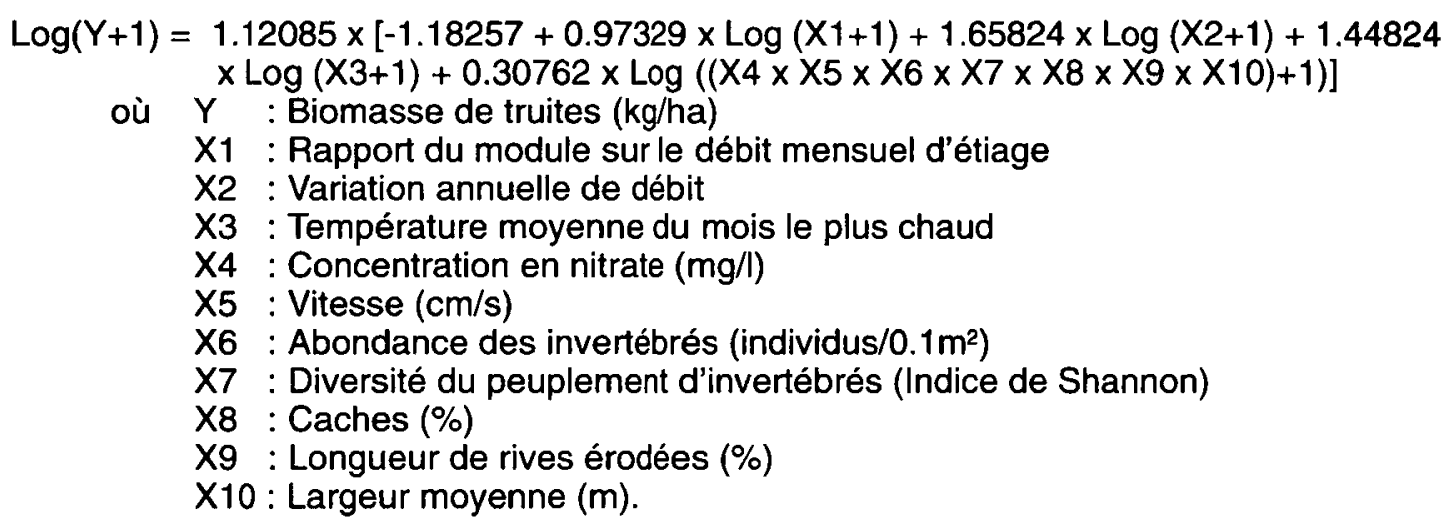

Pour chacune des dix variables, BINNS (1982) propose cinq valeurs de classes de qualité (de 0 à 4) qui sont introduites dans le modèle de calcul.

\section{RÉSULTATS}

Le tableau II présente les caractéristiques d'abondance des truites et les valeurs moyennes des variables de l'habitat. La biomasse maximale est de $3242 \mathrm{~g} / 100 \mathrm{~m}^{2}$, la minimale de $183 \mathrm{~g} / 100 \mathrm{~m}^{2}$. Le coefficient de variation inter-stationnelle est de $69 \%$, alors que le coefficient moyen de variation intra-stationnelle calculé sur 27 stations est de $23,4 \%$. La variation saisonnière de biomasse au sein d'une même station est significativement inférieure $(p<0,01)$ à la variation inter-stationnelle. La densité maximale est de 126 truites $/ 100 \mathrm{~m}^{2}$, la minimale de 5 truites $/ 100 \mathrm{~m}^{2}$. Le coefficient de variation inter-stationnelle est de $96,1 \%$, significativement supérieur $(p<0,01)$ au coefficient moyen de variation intra-stationnelle $(21,8 \%)$ calculé à partir des résultats des quatre campagnes sur 27 des 33 stations étudiées.

\subsection{Application de la méthode du HQI}

Le modèle $n^{\circ 1}$ de la méthode du HQI (BINNS, 1982) a été testé sur les 33 stations (figure 2). Les biomasses théoriques ne sont pas linéairement corrélées aux biomasses observées. Les deux variables doivent être transformées par les logarithmes pour obtenir un ajustement linéaire satisfaisant aux conditions de normalité et d'indépendance des résidus réduits (tableau III). La pente de la droite de régression (0.53) est significativement différente de $1(t=2.56(p<0.01))$. Pour les faibles biomasses, les valeurs théoriques calculées par le modèle sont supérieures aux valeurs observées tandis que les fortes biomasses la situation est inversée. Le plus fort écart est de $2142 \mathrm{~g} / 100 \mathrm{~m}^{2}$, le plus faible de $64 \mathrm{~g} / 100 \mathrm{~m}^{2}$. 
Bull. Fr. Pêche Piscic. (1993) $331: 321-340-327-$

Tableau II : Caractéristiques d'habitat et d'abondance des truites aux différentes stations.

Table II : Habitat features and characteristics of brown trout population for the different sections.

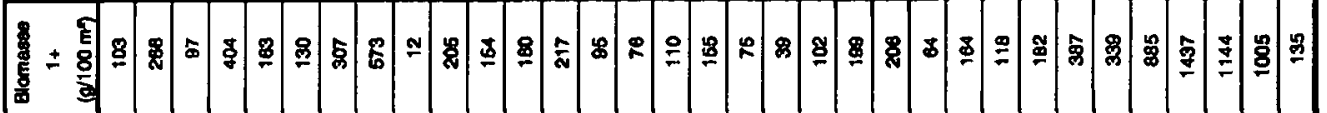

意

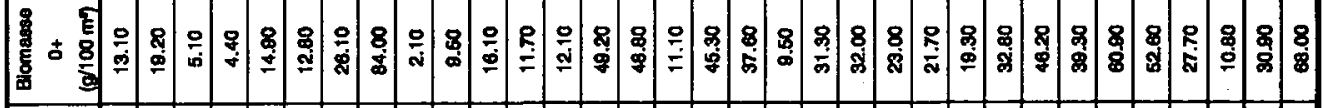

重 直

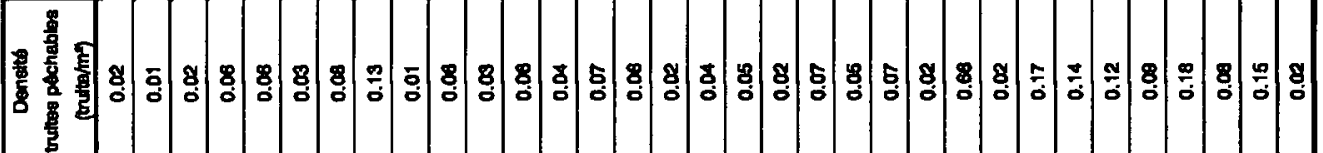

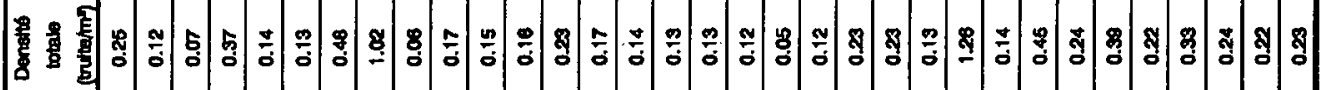

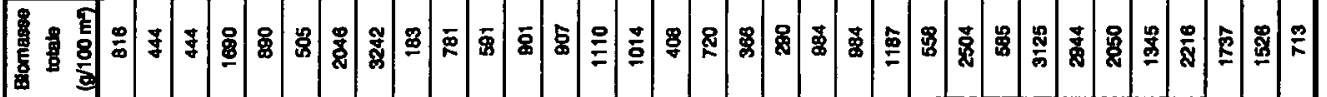

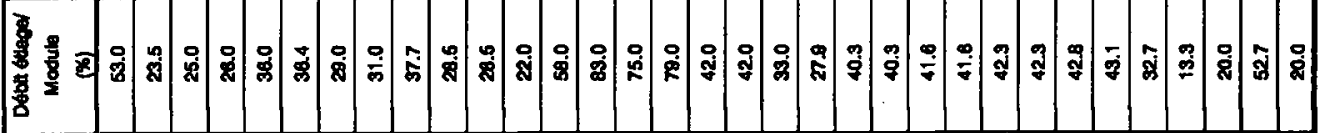

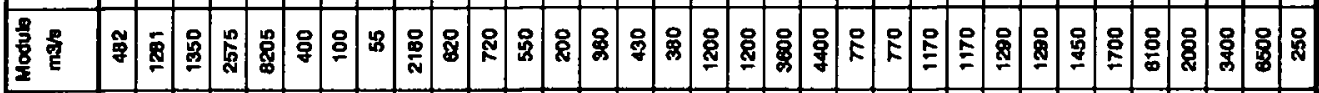

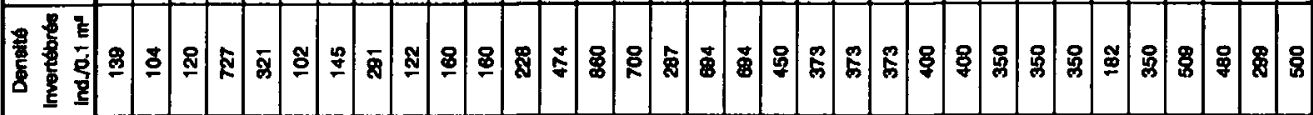

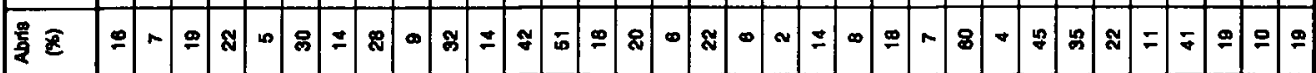

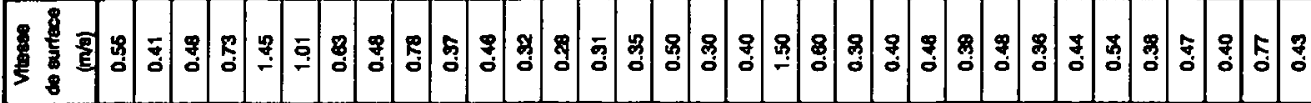

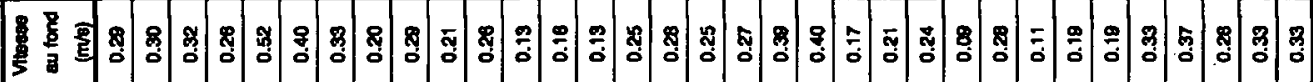

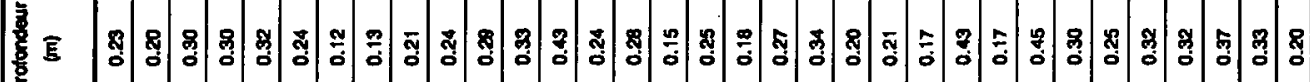

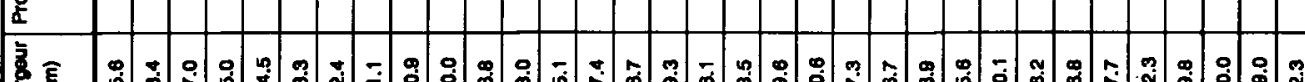

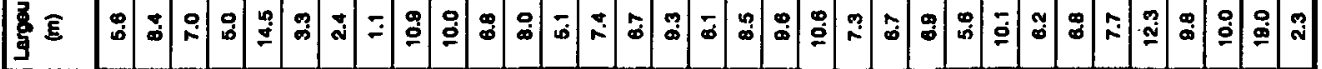

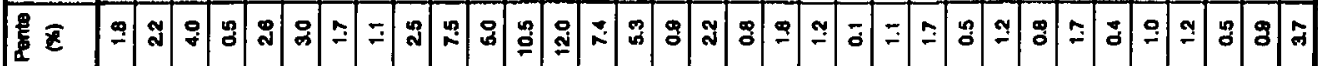

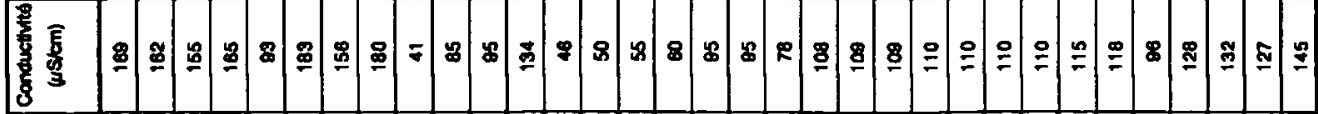

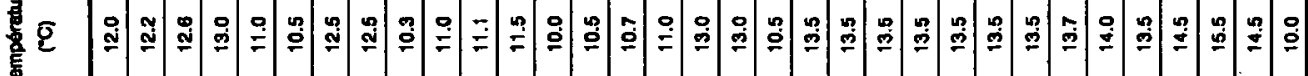

害 E

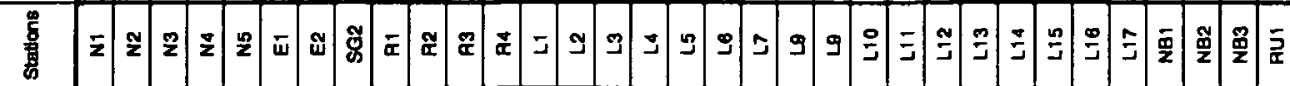


Les résidus réduits de la corrélation ne sont pas significativement corrélés aux densités et biomasses de juvéniles $(0+$ et $1+)$. Seule, la variable abris semble avoir une influence sur les résultats du modèle. On remarque que pour des valeurs de caches inférieures à $10 \%$ équivalant à la classe 0 dans la méthode, les biomasses de truites sont sous-estimées par le modèle. A l'inverse, pour des valeurs de caches comprises entre 10 et $25 \%$ (classe 1), la majorité des biomasses sont surestimées par le modèle. Aucune autre variable de l'habitat n'influence significativement la distribution des résidus du modèle.

Tableau III : Caractéristiques statistiques du modèle linéaire établi entre les biomasses théoriques du modèle HQI et les biomasses observées.

Table III : Statistical characteristics of linear model which related biomass predicted by HQI model to biomass observed.

\begin{tabular}{|c|c|c|c|}
\hline$r^{2}$ & $\begin{array}{c}\text { Statistique d de } \\
\text { Durbin-Watson }\end{array}$ & $\begin{array}{c}\text { Résidus réduits } \\
\text { n'appartenant pas } \\
\text { a l'intervalle }(-1.96+1.96)(\%)\end{array}$ & Dmax \\
\hline 0.64 & 1.65 & 3.03 & 0.0735 \\
\hline
\end{tabular}

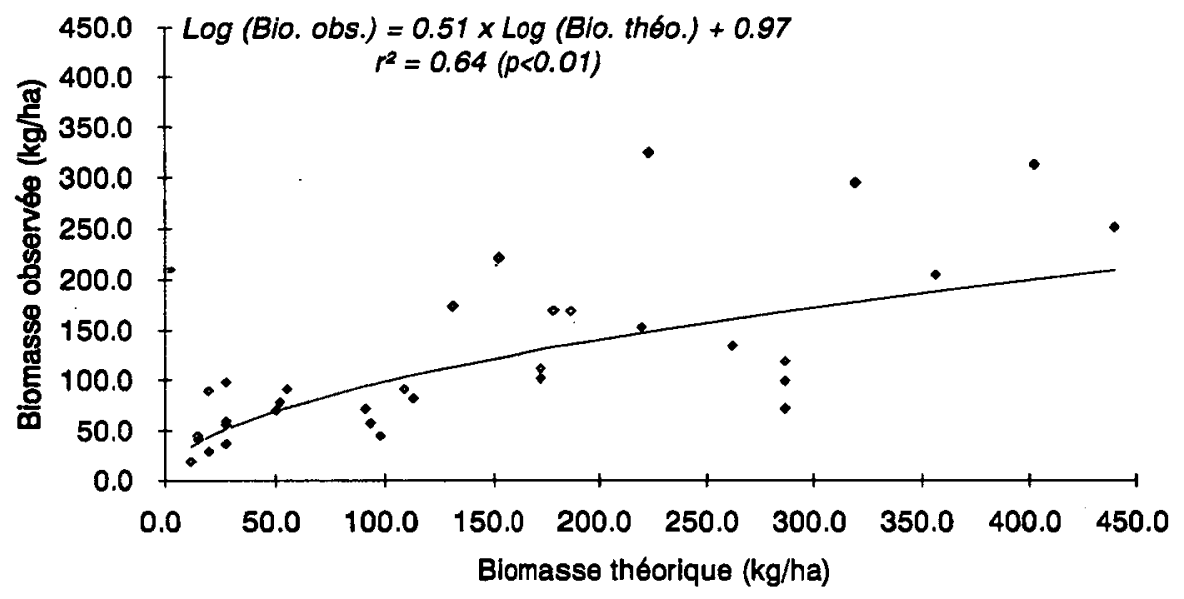

Figure 2 : Relation entre les biomasses observées et les biomasses théoriques : modèle HQI.

Figure 2 : Relationship between biomass observed and biomass predicted : model HQI.

\subsection{Recherche des corrélations entre l'abondance des truites et chaque variable de I'habitat}

\subsubsection{Etude sur les abondances globales}

Pour 5 variables du milieu, des régressions linéaires avec la biomasse totale de truites de la vallée d'Aure (tableau IV, figures 3 à 7) ont pu être établies. Deux seulement appartiennent au modèle $\mathrm{HQl}$ : la température maximale mensuelle et le pourcentage d'abris. Des transformations par les logarithmes ont été nécessaires pour obtenir des modèles linéaires satisfaisant aux conditions requises par les statistiques d de Durbin-Watson $(1.5<d<2.5)$ et $D$ de Kolmogorov-Smirnov (Dmax<0.2307). Ces transformations ont été effectuées pour les variables biomasse, altitude, conductivité, rapport largeur/profondeur et abris. 
Bull. Fr. Pêche Piscic. (1993) $331: 321-340-329-$
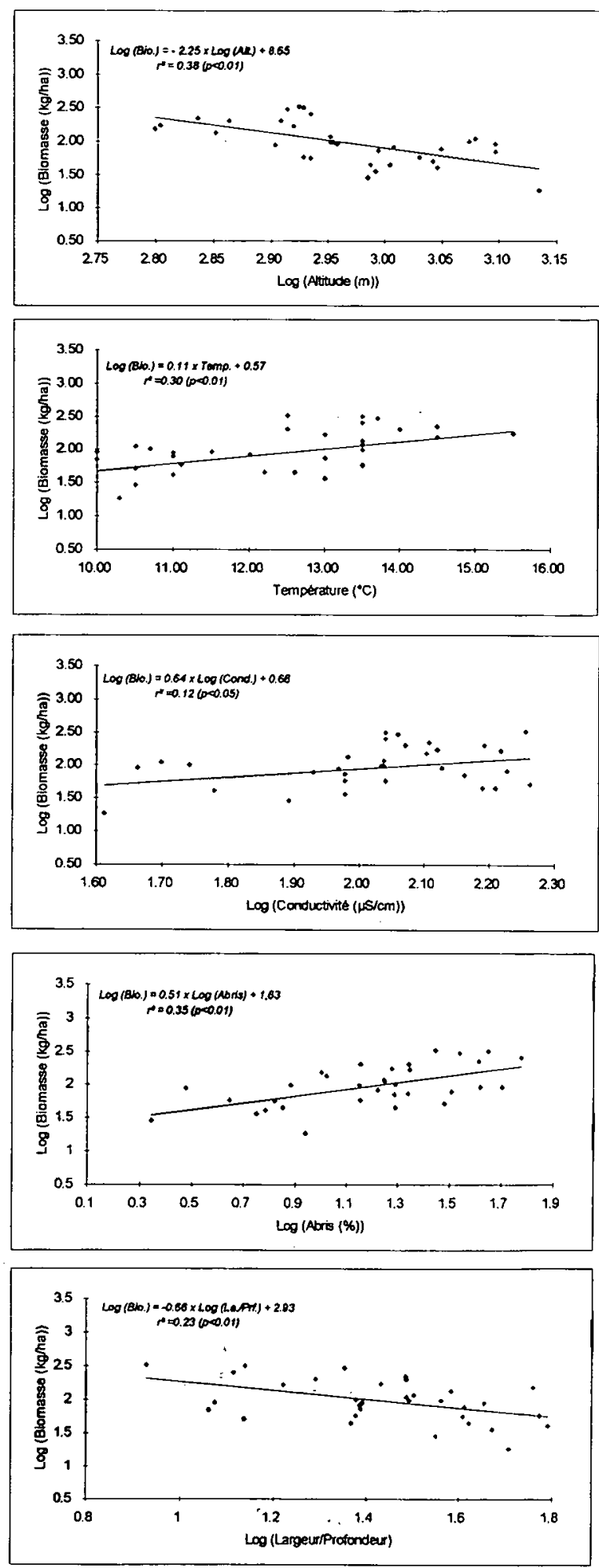

Figures 3 à 7 : Régressions linéaires entre les biomasses totales de truites et les variables d'habitat.

Figures 3 to 7 : Linear regressions between total biomass of trouts and habitat features. 
Tableau IV : Caractéristiques statistiques des régressions linéaires établies entre les biomasses totales de truites et les variables d'habitat.

Table IV : Statistical characteristics of linear regressions which related total biomass to habitat features.

\begin{tabular}{|c|c|c|c|c|}
\hline Variables & $r^{2}$ & $\begin{array}{c}\text { Statistique d de } \\
\text { Durbin-Watson }\end{array}$ & $\begin{array}{c}\text { Résidus réduits } \\
\text { n'appartenant pas } \\
\text { à l'intervalle (-1.96+1.96) (\%) }\end{array}$ & Dmax \\
\hline Log(Altitude) & 0.38 & 1.96 & 0 & 0.074 \\
\hline Temperature & 0.30 & 1.76 & 0 & 0.095 \\
\hline Log(Conductlvité) & 0.12 & 1.54 & 0 & 0.095 \\
\hline Log(Larg./Prf.) & 0.23 & 1.51 & 3 & 0.076 \\
\hline Log(Abris) & 0.35 & 1.52 & 3 & 0.074 \\
\hline
\end{tabular}

Les biomasses totales de truites sont significativement corrélées avec la profondeur moyenne et la viteșse moyenne alors que les densités totales de truites sont significativement corrélées avec huit variables de l'habitat (altitude, température, conductivité, abris, rapport largeur/profondeur, vitesse au fond, pente, largeur) (tableau V).

Tableau V : Coefficient de corrélation de rang de Spearman ( $r s)\left({ }^{*} p<0,05,{ }^{\star \star} p<0,02\right.$, *** $p<0,01)$ établi entre les densités et biomasses totales de truites et les caractéristiques de l'habitat.

Table V : Coefficient of rank correlation of Spearman (rs) $\left(^{*} p<0,05,{ }^{*} p<0,02\right.$, *** $p<0,01$ ) which related total density and total biomass of trouts to habitat features.

\begin{tabular}{|c|c|c|}
\hline & Densité totale & Biomasse totale \\
\hline Variables & rs & rs \\
\hline Altitude & $-0.47^{\star k t}$ & Why \\
\hline Température & $0.38^{*}$ & 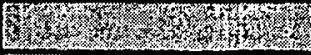 \\
\hline Conductivité & $0.41^{\star \star}$ & 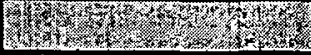 \\
\hline Abris & $0.57^{\star x+k}$ & 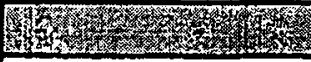 \\
\hline Larg./Prf. & $-0.54^{*+1}$ & $-0.36^{\star}$ \\
\hline Vitesse au fond & -0.41 *t & M \\
\hline Pente & $0.35^{\star}$ & Herly \\
\hline Largeur & $-0.45^{\star \ldots}$ & 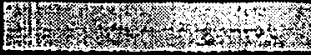 \\
\hline Profondeur & Whay & $0.41^{\star \star}$ \\
\hline
\end{tabular}

\subsubsection{Etude par classe d’âge (tableau VI)}

Les densités et biomasses de truites de l'année $(0+)$ sont significativement corrélées avec les variables altitude, largeur, température et conductivité. II n'existe, par contre, aucune relation avec des variables de l'habitat physique telles que les abris, la profondeur ou la vitesse. Seules les densités et biomasses calculées à partir des échantillonnages d'hiver (février) sont significativement corrélées avec la profondeur moyenne (respectivement $r s=-0.41 \quad(p<0.02)$ et $r s=-0.39(p<0.05))$. Si l'on examine les 
résultats en termes de pourcentages de densités représentés par cette classe d'âge, on note une corrélation avec la profondeur moyenne $(r s=-0.52(p<0.01))$. L'abondance relative des $0+$ dans la population de truites diminue avec l'augmentation de la profondeur moyenne.

Les densités et biomasses de juvéniles $(1+)$ sont corrélées avec l'altitude, la température, la conductivité et les abris. On ne note en revanche aucune relation entre les variables de l'habitat et le pourcentage que représente la classe $1+$ dans l'effectif total.

Les densités de truites de taille supérieure à la taille légale de capture $(180 \mathrm{~mm})$ sont significativement corrélées avec la température, la profondeur moyenne, les abris et l'altitude.

Tableau VI : Coefficient de corrélation de rang de Spearman (rs) ( ${ }^{*} p<0,05,{ }^{* \star} p<0,02$, $\left.{ }^{\star \star \star} p<0,01\right)$ établi entre les densités et biomasses des différentes classes d'âges de truites et les caractéristiques de l'habitat.

Table VI : Coefficient of rank correlation of Spearman (rs) (* $p<0,05,{ }^{* \star} p<0,02$, $\star \star \star \quad p<0,01)$ which related density and biomass of different ageclasses of trouts to habitat features.

\begin{tabular}{|c|c|c|c|c|c|}
\hline & $\begin{array}{c}\text { Densité truites } \\
\text { pêchables }\end{array}$ & $\begin{array}{c}\text { Densité } \\
0+\end{array}$ & $\begin{array}{c}\text { Biomasse } \\
0_{+}\end{array}$ & $\begin{array}{c}\text { Densite } \\
1+\end{array}$ & $\begin{array}{c}\text { Biomässe } \\
1+\end{array}$ \\
\hline Variables & rs & PS & rs & rs & rs \\
\hline Altitude & $-0.68^{\ldots \hbar t}$ & $-0.34^{\star}$ & $-0.38^{\star}$ & $-0.44^{\star \star}$ & $-0.66^{\star \star}$ \\
\hline Température & $0.64^{\star \star \hbar}$ & $0.36^{*}$ & $0.39^{\star}$ & $0.35^{\star}$ & $0.53^{\star k \star}$ \\
\hline Conductivité & (2) & $0.35^{\star}$ & $0.37^{\star}$ & $0.44^{\text {\# }}$ & $0.42^{\star \star}$ \\
\hline Abris & $0.37^{\star}$ & 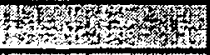 & 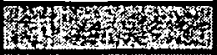 & $0.42^{\text {k }}$ & $0.4^{\star}$ \\
\hline Profondeur & $0.35^{\star}$ & 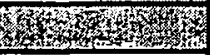 & 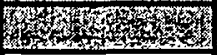 & 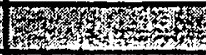 & 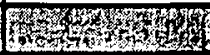 \\
\hline Largeur & 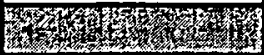 & $0.35^{\star}$ & $0.35^{\star}$ & 第3 & 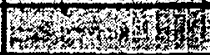 \\
\hline
\end{tabular}

\subsection{Recherche des relations entre l'abondance des truites et les variables de l'habitat à partir de modèles multivariés}

La procédure "pas à pas" a permis de sélectionner cinq variables pour la biomasse totale. Elles expliquent $86 \%$ de la variation de biomasses (figure 8 ). Les cinq variables retenues sont : l'altitude, les abris, le rapport du débit d'étiage sur le module annuel, la largeur et la conductivité.

$\log (Y)=-3.2 \times \log (V 1)-0.43 \times \log (V 2)-0.50 \times \log (V 3)+0.44 \times \log (V 4)+0.32 \times \log (V 5)+11.91$

où $Y=$ Biomasse de truites en $\mathrm{kg} / \mathrm{ha}$

$\mathrm{V} 1$ = Altitude en mètres

$\mathrm{V} 2$ = Conductivité en $\mu \mathrm{S} / \mathrm{cm}$

$\mathrm{V} 3=$ Largeur en mètres

$\mathrm{V} 4=$ Abris en $\%$

V5 = Débit mensuel d'étiage / Module, en \%.

Le tableau VII présente les caractéristiques statistiques du modèle multivarié. Les conditions d'indépendance des résidus (statistique de Durbin-Watson $1.81<\mathrm{d}<2.19$ ) sont satisfaites ainsi que la normalité de la distribution des résidus réduits (statistique de Kolmogorov-Smirnov Dmax<0.2307). 
Tableau VII : Caractéristiques statistiques du modèle multivarié des biomasses totales de truites.

Table VII : Statistical characteristics of multivariate model of total biomass of trouts.

\begin{tabular}{|c|c|c|c|}
\hline$r^{2}$ & $\begin{array}{c}\text { Statistique d de } \\
\text { Durbin-Watson }\end{array}$ & $\begin{array}{c}\text { Résidus réduits } \\
\text { n'appartenant pas } \\
\text { à l'intervalle }(-1.96+1.96)(\%)\end{array}$ & Dmax \\
\hline 0.86 & 2.18 & 0 & 0.1169 \\
\hline
\end{tabular}

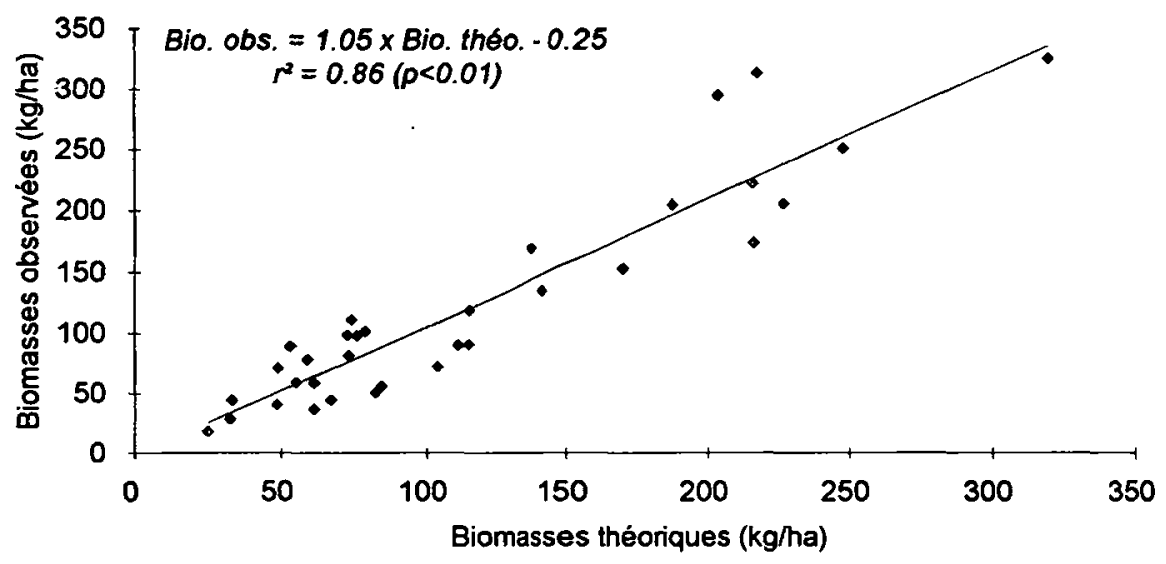

Figure 8 : Relation entre les biomasses observées et les biomasses théoriques : modèle à 5 variables.

Figure 8 : Relationship between biomass observed and biomass predicted : model with 5 variables.

\section{DISCUSSION}

Les populations de truites de la vallée de la Neste d'Aure sont étroitement liées à la qualité physico-chimique de l'eau ainsi qu'à la qualité de l'habitat physique.

\subsection{Test du modèle HQI}

Les biomasses théoriques du modèle HQI ne sont pas linéairement corrélées aux biomasses observées. La relation de type logarithmique mise en évidence dans notre étude indique la pertinence écologique des 10 variables retenues par BINNS et EISERMAN (1979), mais montre un décalage entre la capacité d'accueil tel qu'elle est estimée par le modèle et les valeurs réellement observées sur chacune des 33 stations. CONDER et ANNEAR (1987), SCARNECCHIA et BERGERSEN (1987) ont montré sur d'autres sites que la méthode du HQl permet d'expliquer les variations inter-stationnelles de biomasses. A l'opposé, BOWLBY et ROFF (1986) n'ont pas constaté de corrélation significative entre les biomasses observées et les biomasses théoriques du modèle. Ces résultats contradictoires s'expliquent par le fait que notre étude, comme celles de CONDER et ANNEAR (1987) ainsi que SCARNECCHIA et BERGERSEN (1987), a été conduite sur un site de montagne correspondant au niveau typologique de la zone à truites alors que BOWLBY et ROFF (1986) ont travaillé sur des stations situées en limite aval de la zone à truites. 
Les caractéristiques spécifiques de chaque station (réserve de pêche, débit naturel, débit réservé) ne permettent pas d'expliquer le décalage entre les valeurs observées et les valeurs théoriques. L'abondance des juvéniles n'influence pas non plus de façon significative l'écart entre les deux valeurs de biomasses. On peut également envisager l'hydraulicité comme élément de régulation du recrutement. Toutefois, le modèle HQI intègre dans son calcul deux variables descriptives du régime hydrologique de la rivière (le rapport du débit moyen mensuel d'étiage sur le module et surtout le rapport du débit maximal journalier sur le débit minimal journalier). L'intensité des crues est donc prise en compte dans le calcul, crues qui ont lieu en raison du régime nival au printemps. En fait, seul le potentiel d'abris et plus particulièrement les faibles surfaces de caches semblent avoir une influence sur les résultats du modèle. Un faible potentiel de caches apparaît moins pénalisant pour l'abondance des truites par rapport aux prévisions du modèle. Ce résultat pourrait être attribué à l'estimation des caches et notamment à la sous-estimation des caches occupées par les juvéniles. En effet, la notion de caches et son occupation diffèrent selon la saison, la taille et l'âge du poisson. Ainsi, les juvéniles occuperont des caches de dimension restreinte (de type galets, par exemple) et donc plus difficilement échantillonnables. Toutefois, les stations à faibles surfaces de caches ne possèdent pas un pourcentage de juvéniles significativement supérieur à celui des autres stations. II est important également de souligner que ce modèle a été établi pour des populations de Salmonidés (saumons de fontaines, truites arc-en-ciel et truites communes) vivant en sympathie. Dans le cas de la vallée d'Aure, seule l'espèce truite commune est présente. II n'existe donc pas, à l'inverse des rivières du Wyoming sur lesquelles a été mis au point le modèle $\mathrm{HQI}$, de phénomène de compétition interspécifique pouvant modifier l'occupation de l'habitat.

Ce type de modèle peut donc représenter un outil de gestion intéressant dans la mesure où il permet d'évaluer une capacité d'accueil du milieu. La démarche entreprise dans la méthode semble pertinente du point de vue écologique. En revanche, l'estimation de certaines variables et notamment les caches, ainsi que l'intégration de variables à caractère plus régional pourraient permettre d'améliorer les résultats de cette méthode.

\subsection{Influence des variables physico-chimiques}

La température mensuelle maximale et l'altitude sont liées avec l'ensemble des indices d'abondance de la population de truites (indices globaux ou par classes de taille). Ces deux variables sont significativement corrélées entre elles $\left(r^{2}=0.85(p<0.01)\right)$. Les corrélations mises en évidence entre ces deux facteurs et les caractéristiques de la population de truites ont une signification écologique identique.

La truite est un sténotherme d'eau froide (MILLS, 1971), la température aura une influence directe sur l'ensemble des fonctions physiologiques du poisson. Elle déterminera la répartition géographique tant en altitude (CRISP et al.,1975; SCARNECCHIA et BERGERSEN, 1987) qu'en latitude (RALEIGH et al., 1986). Cette variable peut être considérée comme le facteur limitant universel des populations de truites (BOWLBY et ROFF, 1986). On peut considérer qu'au sein d'une gamme comprise entre 0 et $27^{\circ} \mathrm{C}$ (RICKER, 1934 ; MILLS, 1971 ; MEISNER, 1990), il existe un optimum de croissance et de développement se situant entre 12 et $19^{\circ} \mathrm{C}$ (FROST et BROWN, 1967 ; MILLS, 1971). Ceci conduit à constater des corrélations positives ou négatives avec la température ou l'altitude selon la situation altitudinale des stations étudiées (BURTON et WESCHE, 1974 ; CRISP et al., 1975 ; BOWLBY et ROFF, 1986 ; SCARNECCHIA et BERGERSEN, 1987).

Dans le cadre de notre étude, la gamme des températures maximales mensuelles variant de 10 à $15,5^{\circ} \mathrm{C}$, il est logique de retrouver une corrélation positive avec l'abondance totale des truites (et donc implicitement négative avec l'altitude). Ce sont les basses températures qui constituent le principal facteur limitant pour les biomasses de truites. Au-dessus de $1350 \mathrm{~m}$, la température ne dépasse que très rarement $10^{\circ} \mathrm{C}$, les biomasses de truites sont inférieures à $20 \mathrm{~kg} / \mathrm{ha}$. RICARD et ROQUEPLOT (1976) considèrent que, sur la Neste du Rioumajou, la très faible abondance des truites à des altitudes supérieures à $1300 \mathrm{~m}$ est liée à la température de l'eau. 
En revanche, les relations sont nettement moins fortes avec la densité totale de truites. Pour des valeurs très proches de densités $\left(23\right.$ truites $\left./ 100 \mathrm{~m}^{2}\right)$ et des structures de classes de tailles identiques, les stations situées à $630 \mathrm{~m}$ d'altitude ont une biomasse supérieure de $86 \%$ à celle des stations situées à $1250 \mathrm{~m}$ d'altitude. Cette différence s'explique donc sur la base de variations de croissance des truites. La température influence directement la croissance des truites (ELLIOT, 1984 ; MORTENSEN, 1988 ; BAGLINIĖRE et MAISSE, 1990). Le taux de croissance $(G)$ est directement dépendant de la température (ELLIOT, 1975).

En ce qui concerne les juvéniles et plus particulièrement les $0+$, on note une diminution des densités et biomasses avec l'altitude. II existe donc un gradient d'abondance des juvéniles d'amont vers l'aval. Les pourcentages des cohortes $0+$ et $1+$ ne sont pas liés quant à eux à l'axe amont-aval, à l'inverse de ce qui a été observé sur d'autres rivières en France où le pourcentage des classes d'âges $0+$ et 1+augmente d'aval en amont (NIHOUARN, 1983 ; OMBREDANE et al., 1988 in HAURY et al., 1991 ; BAGLINIĖRE et al., 1989). II n'apparaît pas de ségrégation longitudinale des classes d'âges sur le bassin de la Neste d'Aure. Ceci est directement lié aux pentes des rivières et ruisseaux, ainsi qu'à la présence de barrages infranchissables qui limitent les migrations des truites et notamment la remontée des géniteurs. Ceci semble obliger les poissons à réaliser une grande partie de leur cycle de vie dans un tronçon de rivière de longueur limitée.

La conductivité électrique est la seconde variable de qualité de l'eau corrélée significativement avec la biomasse et la densité de truites. Ces résultats sont comparables à ceux de Mac FADDEN et COOPER (1962) ainsi que SCARNECCHIA et BERGERSEN (1987) qui montrent que la biomasse de truites augmente avec la conductivité de l'eau. Dans le cas de la vallée d'Aure, le calcium contribue de façon déterminante à la conductivité électrique. Or, la concentration en calcium est un facteur limitant pour la croissance des truites. CUINAT (1971) et PHILIPPART (1978) in WELCOMME (1985) établissent d'ailleurs des modèles de calcul des biomasses de truites prenant en compte la concentration en calcium ou l'alcalinité de l'eau.

\subsection{Influence de la qualité de l'habitat physique}

La qualité de l'eau n'est pas le seul facteur permettant d'expliquer les variations de biomasses et de densités de truites dans la vallée d'Aure. L'habitat physique et plus particulièrement les abris, la vitesse au fond, la profondeur, le rapport largeur/profondeur et la pente interviennent de façon significative vis-à-vis de l'abondance des truites.

Les abris représentés par les sous-berges, les sous-blocs, les branchages, les zones turbulentes et les zones profondes (Binns, 1982) sont fortement corrélés avec la biomasse et la densité totale de truites. L'étude par classe de taille révèle en revanche que les densités et biomasses de $0+$ ne sont pas influencées par la quantité d'abris telle qu'elle est estimée dans la méthodologie. Ceci s'explique par la difficulté à quantifier les abris utilisés par les juvéniles (galets et petits blocs). A l'inverse, l'abondance des truites de taille supérieure à la taille légale de capture $(180 \mathrm{~mm})$ est fortement corrélée à l'abondance des abris. Les truites adultes utilisent des abris de taille plus importante ainsi que des zones profondes plus marquées.

De nombreux auteurs considèrent les abris comme une des variables de l'habitat physique les plus déterminantes vis-à-vis de l'abondance des truites (LEWIS, 1969 ; HUNT, 1976 ; ENK, 1977 ; FAUSH et WHITE, 1981 ; BOVEE, 1982 ; CUNJAK et POWER, 1986 ; GATZ et al., 1987). NIELSEN (1986) montre que l'abondance des abris peut expliquer de 33 à $74 \%$ de la variation de densités de truites. L'augmentation du nombre d'abris entraîne une augmentation de la biomasse de truites (BOUSSU, 1954 ; HUNT, 1976 ; NASLUND, 1989). WESCHE et al. (1987) indiquent une corrélation significative entre la surface occupée par les sous-berges et le nombre de truites. II établit deux modèles de calcul des biomasses de truites basés sur la quantité d'abris et la profondeur de la station.

Le rapport largeur/profondeur est significativement corrélé avec les abris $\left(r^{2}=0,64\right.$ $(p<0.01))$ et plus particulièrement avec les zones profondes. Sa signification écologique 
vis-à-vis de l'abondance des truites sera donc discutée en termes d'abris et d'importance de la profondeur de l'eau. On notera que les auteurs ayant utilisé cette variable (MORTENSEN, 1977 ; SCARNECCHIA et BERGERSEN, 1987) trouvent également une corrélation significative avec la biomasse de truites.

La vitesse et la profondeur interviennent sur l'abondance des truites par l'intermédiaire de leur rôle prépondérant dans le choix du microhabitat qui correspond à la position exacte de la truite à un instant donné et pour un type d'activité donnée (nutrition, reproduction) (BALDES et VINCENT, 1969 ; SHIRVELL et DUNGEY, 1983). La vitesse sera considérée comme le principal facteur intervenant dans le choix des sites de nutrition (SHIRVELL et DUNGEY, 1983 ; BACHMAN, 1984 ; DEGRAAF et BAIN, 1986 ; HEGGENES et SALTVEIT, 1990). Les corrélations entre l'abondance des truites et les valeurs moyennes de ces deux variables mises en évidence dans notre étude, comme dans beaucoup d'autres (LEWIS, 1969 ; WESCHE, 1976 ; BOLHIN, 1977 ; KROG et HERMANSEN, 1986 ; NIELSEN, 1986), doivent être interprétées en termes de quantité et de qualité de microhabitats disponibles dans chaque station. En effet, la truite commune possède des exigences strictes vis-à-vis de ces deux variables, exigences qui ont pu être traduites par des courbes de préférenda (BOVEE, 1982 ; RALEIGH et al., 1986 ; FRAGNOUD, 1987 ; BELAUD et al., 1989 ; CHAVEROCHE, 1990). En comparant les valeurs des préférenda et la disponibilité des trois variables mesurées dans le milieu au sein de cellules hydrodynamiques homogènes, la méthode des "microhabitats" (méthode IFIM (BOVEE, 1982)), permet de calculer une Surface Pondérée Utile (SPU) pour différents stades de développement des truites. Elle permet de juger de l'adéquation des conditions morphodynamiques par rapport aux exigences du poisson (SOUCHON et 'al., 1989). Cette méthode admet que la population de truites sera limitée par les conditions de vitesse, de profondeur et de granulométrie. STALNAKER (1979), LOAR et al. (1985), SOUCHON et al. (1989) trouvent une corrélation entre l'abondance des truites et la Surface Pondérée Utile. A l'inverse, SHIRVELL et MORANTZ (1983), CONDER et ANNEAR (1987) et CHAVEROCHE (1990) ne mettent pas en évidence de corrélation entre cette Surface Pondérée Utile et les biomasses et densités de truites. Le rôle de ces variables qui paraît évident dans le choix du microhabitat reste malgré tout plus difficile à appréhender vis-à-vis de l'abondance des truites. On peut également souligner que l'influence de ces différents facteurs varient en fonction du stade de développement. La profondeur est positivement corrélée avec la biomasse totale ou la densité de truites pêchables mais négativement corrélée avec la densité de $0+$ en hiver. Ces observations confirment le fait que les juvéniles utilisent souvent des zones de faible profondeur (LINDROTH, 1955 ; BAGLINIĖRE et CHAMPIGNEULLE, 1982 in HAURY et al., 1991 ; FRAGNOUD, 1987 ; HEGGENES, 1988). Toutefois, hormis pour cette situation hivernale, il est difficile de mettre en évidence des relations entre les variables profondeur ou vitesse moyenne et l'abondance des $0+$ et $1+$. Ceci peut s'expliquer par le choix de l'échelle d'étude qui correspond à la séquence de macrohabitat. II apparaît, en effet, qu'une étude détaillée par faciès d'écoulement permettrait probablement de mieux cerner la ségrégation spatiale des différentes classes d'âges. L'échelle de la séquence ne constitue donc pas un outil intéressant du point de vue de l'étude de la ségrégation spatiale des différentes cohortes de la population de truites.

La pente et la largeur sont significativement corrélées à la densité de truites. LINDROTH (1955), BAGLINIĖRE et ARRIBE-MOUTOUNET (1985) montrent que la largeur intervient sur l'abondance des truites par l'intermédiaire de l'effet de berge. Ces dernières jouent un rôle au niveau des abris et des conditions d'écoulement. On remarquera que les ruisseaux de faibles largeurs $(<3 \mathrm{~m})$ de la vallée d'Aure situés à moins de $1000 \mathrm{~m}$ d'altitude possèdent des densités de truites supérieures à 20 truites $/ 100 \mathrm{~m}^{2}$. En ce qui concerne la pente, KOZEL et al. (1990) mettent en évidence une corrélation négative entre cette variable et la densité de truites. II distingue deux groupes de stations en fonction de leur pente respective. On peut considérer que ce facteur joue un rôle vis-à-vis des déplacements de poissons. De fortes pentes limiteront les possibilités de migration et donc l'espace disponible pour une population de truites.

L'abondance et la diversité des invertébrés ne sont pas directement corrélées à la biomasse et à la densité de truites. Ces deux descripteurs utilisés dans le modèle HQI ne 
permettent pas d'analyser le niveau de relation trophique existant entre la macrofaune benthique et les populations de truites. La production annuelle constituerait probablement un meilleur indice puisqu'elle intègre à la fois l'abondance pondérale et numérique de la faune benthique ainsi que la durée des cycles de vie des insectes. Il est important également de signaler que la dérive des invertébrés benthiques ne constitue pas la totalité du régime alimentaire de la truite qui, en certaines périodes de l'année, peut utiliser les apports exogènes à partir des berges (NEVEU, 1991). En ce qui concerne le débit, on constate que la variable rapport débit d'étiage/module intervient dans le modèle multivarié de biomasse. Cette variable dont les caractéristiques sont également utilisées dans la méthode du HQI, bien que n'ayant aucune corrélation significative avec la biomasse ou la densité de truites, est susceptible d'apporter une information supplémentaire vis-à-vis de l'abondance des poissons lorsqu'elle est associée à un ensemble d'autres variables de l'habitat. En ce qui concerne l'intensité des crues, la variable débit maximal journalier/débit minimal journalier n'est corrélée avec aucune des variables d'abondance de la population de truites. La densité et la biomasse des $0+$ qui sont en général les plus vulnérables vis-à-vis des crues, ne sont pas liées à la variation maximale de débit dans une année. II est important de signaler que les années d'étude ont été des années de faible hydraulicité avec un enneigement réduit induisant de faibles crues printanières. De plus, l'ensemble du bassin versant possède un régime hydrologique très semblable.

Aucun des facteurs pris indépendamment ne permet d'expliquer de façon satisfaisante les variations inter-stationnelles d'abondances de truites. II est donc nécessaire d'utiliser plusieurs variables du milieu pour expliquer les variations de biomasses de truites sur la Neste d'Aure et ses affluents. Les résultats des régressions multiples "pas à pas" montrent bien que cet indice est lié à la fois à des variables physico-chimiques mais également à des variables de l'habitat physique et du débit. Les modèles statistiques de calcul de biomasses de truites disponibles dans la littérature (CUINAT, 1971 ; WESCHE, 1976 ; BINNS et EISERMAN, 1979 ; RALEIGH et al., 1986 ; LANKA et al., 1987) utilisent de 3 à 18 variables du milieu. Cependant, il est important de signaler que ces modèles donnent souvent une image ponctuelle de la capacité d'accueil du milieu. Ils intègrent peu les problèmes de recrutement (habitat des alevins), de reproduction (habitat de reproduction disponible) ainsi que les évènements antérieurs (crues, vidanges, pollutions accidentelles) susceptibles de déstabiliser la population de truites. Ainsi, on peut noter dans notre étude le faible niveau de relation des truites de l'année $\left(0_{+}\right)$avec les facteurs physiques (à l'exception de la profondeur), démontrant ainsi le rôle probable d'autres paramètres parmi lesquels on pourrait citer ceux liés à l'habitat de reproduction (granulométrie, vitesse, profondeur). De plus, la mise en place de ces modèles impose implicitement de considérer qu'à l'intérieur des sites d'étude choisis, la biomasse en place soit une image fidèle de la capacité d'accueil. Seules, des expériences de modifications de cette capacité d'accueil (ajouts d'abris, augmentation de débit) permettraient de vérifier les réponses de la population de truites et donc le véritable niveau de relation qu'elle entretient avec le milieu (FAUSH et al., 1988).

\subsection{Application à la gestion des cours d'eau}

La connaissance des besoins et du comportement de la truite fario doivent impérativement être pris en compte dans l'aménagement et la gestion des cours d'eau (HEGGENES, 1988). Définir la capacité d'accueil de la rivière, tant du point de vue de l'espace mais également du point de vue du réseau trophique, doit constituer l'élément de base de la gestion des ressources trutticoles. L'étude des relations entre les caractéristiques du milieu et l'abondance pondérale et numérique des populations de truites menée dans la vallée de la Neste d'Aure permet de mettre en évidence un certain nombre de facteurs limitants. Les différentes corrélations entre les variables du milieu et les biomasses de truites se proposent de mieux définir les bases de cette capacité d'accueil.

L'extension des résultats obtenus pourrait, dans un premier temps, se limiter aux rivières pyrénéennes dont il faudrait par ailleurs établir une typologie fonctionnelle, tant du point de vue des peuplements piscicoles que des habitats. La validation et l'amélioration de 
la démarche et des outils obtenus doivent passer par des tests sur d'autres sites, étape indispensable dans l'élaboration de modèle statistique de calcul de biomasses (FAUSH et al., 1988), mais également sur une étude plus détaillée de la ségrégation spatiale entre les différentes classes d'âges à l'échelle du macrohabitat.

\section{REMERCIEMENTS}

Les auteurs tiennent à remercier pour leur aide et leur collaboration : E.D.F. (Unité Energie Midi-Pyrénées), la Fédération de Pêche des Hautes-Pyrénées et plus particulièrement son conseiller scientifique, Monsieur Noël $A B A D$, ainsi que la Société de Pêche la Gaule Louronnaise et son Président Monsieur Jean-Marie BAPEL.

\section{BIBLIOGRAPHIE}

BACHMAN R.A., 1984. Foraging behaviour of free-ranging wild and hatchery brown trout in a stream. Trans. Am. Fish. Soc., 113 (1), 1-32.

BAGLINIERE J.L., ARRIBE-MOUTOUNET D., 1985. Microrépartition de populations de truites communes (Salmo trutta L.), de juvéniles de saumon atlantique (Salmo salarL.) et des autres espèces présentes dans la partie haute du Scorff (Bretagne). Hydrobiologia., 120, 229-239.

BAGLINIERE J.L., MAISSE G., LEBAIL P.Y., NIHOUARN A., 1989. Population dynamics of brown trout, Salmo trutta L., in a tributary in Brittany (France) : spawning and juveniles. J. Fish. Biol., 34, 97-110.

BAGLINIERE J.L., MAISSE G., 1990. La croissance de la truite commune (Salmo trutta L.) sur le bassin du Scorff. Bull. Fr. Pêche Piscic., 318, 89-101.

BALDES R.J., VINCENT R.E., 1969. Physical parameters of microhabitats occupied by brown trout in an experimental flume. Trans. Am. Fish. Soc., 98 (2), 230-238.

BELAUD A., CHAVEROCHE P., LIM P., SABATON C., 1989. Probability-of-use curves applied to brown trout (Salmo trutta L.) in rivers of Southern France. Regulated Rivers : Research and management, $\mathrm{Vol} 3,321-336$.

BINNS N.A., EISERMAN F.M., 1979. Quantification of fluvial trout habitat in Wyoming. Trans. Am. Fish. Soc., 108 (3), 215-228.

BINNS N.A., 1982. Habitat Quality Index : procedure manual. Wyoming Game and Fish Department. 209 p.

BOLHIN T., 1977. Habitat selection and intercohort competition of juvenile sea-trout Salmo trutta. Oïkos, 29, 112-117.

BOUSSU M.F., 1954. Relationship between trout populations and cover on a small stream. J. Wild Manag., 18 (2), 229-239.

BOVEE K.D., 1982. A guide to stream habitat analysis using Instream Flow Incremental Methodology. Instream Flow Information Paper $n^{\circ} 12$, FWS/OBS 82/86, $248 \mathrm{p}$.

BOWLBY J.N., ROFF J.C., 1986. Trout biomass and habitat relationship in Southern Ontario streams. Trans. Am. Fish. Soc., 115, 503-514.

BURTON R.A., WESCHE T.A., 1974. Relationship of duration of flows and selected watershed parameters to the standing crop estimates of trout populations. University of Wyoming, Water Resources Research Institute, Water Resources Series Number 52, Laramie.

BUTLER R.L., HAWTHORNE V.M., 1968. The reactions of dominant trout to changes in overhead artificial cover. Trans. Am. Fish. Soc., 97, 37-41.

CHAVEROCHE P., 1990. Recherche sur les préférences d'habitat de la truite fario (Salmo trutta L.) (Aide à la détermination de la valeur d'un débit réservé). Thèse de Docteur en Sciences, Université de Provence, Marseille, 214 p. 
CONDER A.L., ANNEAR T.C., 1987. Test of weighted usable area derived from a PHABSIM model for instream flow studies on trout streams. N. Am. J. Fish. Manage., 7, 339-350.

CRISP D.T., MANN R.H.K., McCORMACK J.C., 1975. The populations of fish in the River Tees system on the Moor House National Nature Reserve, Westmorland. J. Fish. Biol., 7, 573-593.

CUINAT R., 1971. Principaux caractères démographiques observés sur 50 rivières à truites françaises. Influence de la pente et du calcium. Ann. Hydrobiol., 2 (2), 187-207.

CUNJAK R.A., POWER G., 1986. Winter habitat utilization by stream resident brook trout (Salvelinus fontinalis) and brown trout (Salmo trutta). Can. J. Fish Aquat. Sci., 43, 1970-1971.

DAGNELIE P., 1975. Analyses statistiques à plusieurs variables. Presses agronomiques de Gembloux, Gembloux, $362 p$.

DE LURY D.B., 1951. On the planning of experiments for estimation of fish populations. J. Fish. Res. Board. Can., 8, 281-307.

DEGRAAF D.A., BAIN L.H., 1986. Habitat use by and preferences of juveniles Atlantic salmon in two Newfoundland rivers. Trans. Am. Fish. Soc., 115, 671-681.

EIFERT W.H., WESCHE T.A., 1982. Evaluation of the stream reach inventory and channel stability index for instream habitat analysis. Water Resources Series, 82, $97 \mathrm{p}$.

ELLIOT J.M., 1975. The growth rate of brown trout, Salmo trutta L, fed on maximum rations. J. Anim. Ecol., 44, 805-821.

ELLIOT J.M., 1984. Growth, size, biomass and production of young migratory trout Salmo trutta in a Lake District stream. J. Anim. Ecol., 53, 979-994.

ENK M.D., 1977. Instream overhead bank cover and trout abundance in two Michigan streams. M.S. Thesis. Mich. State Univ. East Langing, 127 p.

FAUSH K.D., WHITE R.J., 1981. Competition between brook trout (Salvelinus fontinalis) and brown trout (Salmo trutta) in a Michigan stream. Can. J. Fish. Aquat. Sci., 38, 1220-1227.

FAUSH K.D., HAWKES C.L., PARSONS M.G., 1988. Models that predict the standing crop of stream fish from habitat variables. U.S. Forest Service General Technical Report PNW-GTR - 213.

FRAGNOUD E., 1987. Préférence d'habitat de la truite fario (Salmo trutta fario L. 1758) en rivière. Thèse de Docteur en Sciences. Laboratoire Hydroécologie Quantitative. C.E.M.A.G.R.E.F., Lyon, 435 p.

FROST W.E., BROWN M.E., 1967. The trout. Collins, St James Place, London, 286 p.

GATZ Jr, SALE A.J., LOAR J.M., 1987. Habitat shifts in rainbow trout : competitive influence of brown trout. Oecologia, 74, 7-19.

HARRIS D.D., HUBERT W.A., WESCHE T.A., 1991. Brown trout population and habitat response to enhanced minimum flow in Douglas Creek, Wyoming. Rivers, 2 (4), 285-294.

HAURY J., OMBREDANE D., BAGLINIERE J.L:, 1991. L'habitat de la truite commune (Salmo trutta L.) en eaux courantes. In BAGLINIERE J.L., MAISSE G., La truite, biologie et écologie, 47-96, INRA Publ., Paris.

HEGGENES J., 1988. Physical habitat selection by brown trout (Salmo trutta) in riverine systems. Nordic. J. Freshw. Res., 64, 74-90.

HEGGENES J., SALTVEIT S.J., 1990. Seasonal and spatial microhabitat selection and segregation in young Atlantic salmon, Salmo salar L., and brown trout, Salmo trutta L., in a Norwegian river. J. Fish. Biol., 36, 707-720.

HUNT R.L., 1976. A long-term evaluation of trout habitat development and its relation to improving management-related research. Trans. Am. Fish. Soc., 105 (3), 361-364. 
KROG G., HERMANSEN H., 1986. Physical structure and brown trout (Salmo trutta fario) populations in small Danish lowland. Fish. Magt., 17 (4), 216-222.

KOZEL S.J., HUBERT W.J., PARSONS G.P., 1990. Habitat features and trout abundance relative to gradient in some Wyoming streams. Northwest Science, 63 (4), 175-182.

LANKA R.P., HUBERT W.A., WESCHE T.A., 1987. Relationships of geomorphology to stream habitat and trout standing stock in small rocky mountain streams. Trans. Am. Fish. Soc., 116, 21-28.

LAURENT M., LAMARQUE P., 1975. Utilisation de la méthode des captures successives (De Lury) pour l'évaluation des peuplements piscicoles. Bull. Fr. Piscic., 259, 66-67.

LEWIS S.L., 1969. Physical factors influencing fish populations in pool of a trout stream. Trans. Am. Fish. Soc., 115, 183-195.

LINDROTH A., 1955. Distribution territorial behavior and movements of sea trout fry in the river Indalsälven. Rep. Int. Freshwater Res. Drottningholm., 36, 104-119.

LOAR J.M., SALE M.J., CADA G.F., 1985. Application of habitat evaluation in southern Appalachian trout stream. Environmental Sciences Division publication, 2383, ORNL/TM. 9323, 230 p.

McFADDEN J.T., COOPER E.L., 1962. An ecological comparison of six populations of brown trout (Salmo trutta). Trans. Am. Fish. Soc., 91, 53-62.

MEISNER D.J., 1990. Potential loss of thermal habitat for brook trout, due to climatic warning, in two Southern Ontario streams. Trans. Am. Fish. Soc., 119, 282-291.

MILLS D., 1971. Salmon and trout : a resource, its ecology, conservation and management. New York, St. Martin's Press, $351 \mathrm{p}$.

MORTENSEN E., 1977. The population dynamics of young trout (Salmo trutta L.) in a Danish brook. J. Fish. Biol., 10, 23-33.

MORTENSEN E., 1988. The significance of temperature and food as factors affecting the growth of brown trout, Salmo trutta L., in four Danish streams. Pol. Arch. Hydrobiol., $35(3 / 4), 533-544$.

NASLUND I., 1989. Effects of habitat improvement on the brown trout Salmo trutta L., population of a northern Swedish stream. Aquaculture and Fisheries Management, $20,463-474$.

NEVEU A., 1991. Stratégie alimentaire de la truite commune (Salmo trutta L.) en eaux courantes. In BAGLINIERE J.L., MAISSE G., La truite, biologie et écologie, 97-120, INRA Publ., Paris.

NIELSEN G., 1986. Dispersion of brown trout (Salmo trutta L.) in relation to stream cover and water depth. Pol. Arch. Hydrobio., 33 (3/4), 475-488.

NIHOUARN A., 1983. Etude de la truite commune (Salmo trutta L.) dans le bassin du Scorff (Morbihan) : démographie, reproduction, migrations. Thèse de 3ème cycle Ecologie, Univ. Rennes 1., 64 p.

RALEIGH R.F., ZUCKERMAN L.D., NELSON P.C., 1986. Habitat suitability index models and stream flow suitability curves: brown trout, revised. U.S. Fish. Wild. Ser. Biol. Rep., 82 (10-124), 65 p.

RICARD J.M., ROQUEPLOT C., 1976. Etude sur la dynamique des populations de truites et les aménagements piscicoles du Rioumajou. Thèse Docteur en Sciences, Institut National Polytechnique de Toulouse, $172 \mathrm{p}$.

RICKER W., 1934. An ecological classification of certain Ontario streams. University of Toronto studies, Biological Series 37. Publication of Ontario Fisheries Research Laboratory 49, 7-114.

SCARNECCHIA D.L., BERGERSEN E.P., 1987. Trout production and standing crop in Colorado's small streams, as related to environmental features. N. Am. J. Fish. Manage., 7, 315-330. 
SHIRVELL C.S., DUNGEY R.G., 1983. Microhabitats chosen by brown trout for feeding and spawning in rivers. Trans. Am. Fish. Soc., 112, 355-367.

SHIRVELL C.S, MORANTZ D.L., 1983. Assessments of instream flow incremental methodology for Atlantic salmon in Nova Scotia. Trans. Can. Elect. Assoc., vol 22, 83-H-108, 22 p.

SOUCHON Y., TROCHERIE F., FRAGNOUD E., LACOMBE C., 1989. Les modèles numériques des microhabitats des poissons : applications et nouveaux développements. Rev. Scie. Eau., 2, 807-830.

SPRENT P., 1992. Pratique des statistiques non-paramétriques. INRA éditions, Paris, $294 \mathrm{p}$.

STALNAKER C., 1979. The use of habitat structure preferenda for establishing flow regimes necessary for maintenance of fish habitat. In WARD et STANDFORD, The ecology of regulated streams, 321-337, New York, Plenum.

TOMASSONE R., LESQUOY E., MILLER C., 1983. La régression, nouveaux regards sur une ancienne méthode statistique. INRA actualités scientifiques et agronomiques, 13, Paris, $177 \mathrm{p}$.

TOMASSONE R., DERVIN C., MASSON J.P., 1993. Biométrie. Modélisation de phénomènes biologiques. Masson, Paris, $553 p$.

WELCOMME R.L., 1985. River fisheries. F.A.O. Fish. Tech. Pap., 262, 330 p.

WESCHE T.A., 1976. Development and application of a trout cover rating system for IFN determinations. In Instream Flow Needs, vol II, 224-234.

WESCHE T.A., GOERTLER C.M., FRYE C.B., 1987. Contribution of riparian vegetation to trout cover in small streams. N. Am. J. Fish. Manage., 7, 151-153. 